Cahiers d'études italiennes

1 | 2004

NOVECENTO... E DINTORNI

Dire la guerre?

\title{
Due uomini in piedi sul greto di un fiume
}

\section{Tommaso Santi}

\section{OpenEdition}

\section{Journals}

Edizione digitale

URL: http://journals.openedition.org/cei/974

DOI: $10.4000 /$ cei.974

ISSN: 2260-779X

\section{Editore}

UGA Éditions/Université Grenoble Alpes

\section{Edizione cartacea}

Data di pubblicazione: 15 novembre 2004

Paginazione: 216-225

ISBN: 978-2-84310-057-4

ISSN: 1770-9571

Notizia bibliografica digitale

Tommaso Santi, «Due uomini in piedi sul greto di un fiume», Cahiers d'études italiennes [Online], 1 | 2004, online dal 15 mai 2006, consultato il 27 mars 2021. URL: http://journals.openedition.org/cei/ 974 ; DOI: https://doi.org/10.4000/cei.974 


\title{
DUE UOMINI IN PIEDI
}

SUL GRETO DI UN FIUME

\author{
Tommaso Santi
}

\begin{abstract}
Due uomini sono in mezzo a un fiume, con i piedi nell'acqua ferma e limacciosa, sovrastati da un cielo plumbeo. Walter, il più anziano, ha in dosso un vestito di buon taglio, ma ormai logoro, e con un binocolo guarda un punto lontano davanti a sé. Carlo, il più giovane, è a torso nudo e ha un cappello da pescatore in testa. Anche lui ha un binocolo, ma lo tiene a tracolla e non guarda niente.
\end{abstract}

WALTER - Ci siamo...

CARLO. - Non mi va di restare qui.

W. - Dai, zitto, magari sentiamo qualcosa. C’è la sigla...

C. - Potevamo stare a casa. Abbiamo i piedi nel fango, non mi piace.

W. - Io voglio vedere la tv. Il signor Bi ha una bellissima televisione, qui abbiamo una splendida visuale e io voglio godermi in santa pace i programmi...

C. - Non si capisce nemmeno quello che dicono.

W. - Siamo nella civiltà dell'immagine.

C. - Ah sì...

W. - La parola è marginale, tutto è costruito in funzione... (Si interrompe bruscamente) Inizia, inizia... guarda anche tu, dai...

C. - Mi fanno male i piedi: te lo ripeto ogni volta, tu continui a trascinarmi in mezzo a questo fiume puzzolente.

W. - Non sono io che ti trascino, sei tu che vuoi venire a vedere la tv.

C. - Va bene, interessa anche a me, ma adesso basta. Si sta male, possibile che non te ne renda conto: guarda come siamo ridotti. Ogni giorno la stessa storia.

W. -E non ci pensare... (Comincia a ridere)Oh, ma lo vedi? Quel conduttore lì è fantastico, mi fa morire: ha tirato... ha tirato l'elastico delle mutandine della ballerina, e ci ha rovesciato dentro un gelato... un cono! Ci crederesti, tu?

C. - Fa caldo. Mai stato un giugno così caldo.

W. -... 'spetta 'spetta... nooo! Prendi il canocchiale, questo lo devi vedere... 


\title{
DEUX HOMMES DEBOUT
}

SUR LA GRÈVE D'UN FLEUVE

\author{
Tommaso Santi \\ Traduction française de Christophe Mileschi, revue par l'auteur
}

\begin{abstract}
Deux hommes se trouvent au milieu d'une rivière, les pieds dans l'eau immobile et vaseuse, sous un ciel de plomb. Walter, le plus vieux, porte un habit bien coupé, mais usé, et avec une paire de jumelles il regarde un point situé au loin devant lui. Carlo, le plus jeune, est torse nu et il a un chapeau de pêcheur sur la tête. Lui aussi a des jumelles, mais il les tient en bandoulière et ne regarde rien.
\end{abstract}

WALTER - Ça y est...

CARLO - J'ai pas envie de rester là.

W. - Allez, tais-toi, on va peut-être entendre quelque chose. Ah, c'est le gingle...

C. - On aurait pu rester à la maison. On a les pieds dans la boue, j’aime pas ça.

W. - Moi, je veux regarder la télé. Monsieur Bé a un très beau téléviseur, d'ici on a une vue splendide, et je veux avoir la paix pour profiter tranquillement des programmes...

C. - On ne comprend même pas ce qu'ils disent.

W. - On est dans la civilisation de l'image.

C. - Ah c'est vrai...

W. - Les mots sont une question marginale, tout est construit en fonction de... (Il s’interrompt brutalement). Ça commence, ça commence... toi aussi regarde, allez...

C. - J'ai mal aux pieds : je te le dis à chaque fois, tu t'acharnes à m'obliger à venir au milieu de cette rivière puante.

W. - Ce n'est pas moi qui t'oblige à venir, c'est toi qui veux venir voir la télé.

C. - D'accord, moi aussi ça m'intéresse, mais maintenant ça suffit. On n'est pas bien, comment tu fais pour ne pas t'en rendre compte: regarde dans quel état on est. Tous les jours c'est la même histoire.

W. - Pense à autre chose... (Il commence à rire). Oh, mais t'as vu? Cet animateur est génial, il est trop : il a tiré... il a tiré sur l'élastique de la petite culotte de la danseuse, et il fourré une glace dedans... un cône! 
C. - (Svogliatamente prende il binocolo e guarda) Che è?

W. - Il mago! Pazzesco!

C. - Ha tagliato in due l'aiutante...

W. - (Pieno di ammirazione) Incredibile...

C. - L'ha tagliata in due per davvero!

W. - (C.s.) E un grande...

Buio. La luce sale lentamente, Carlo e Walter sono sempre in mezzo al fiume, il livello dell'acqua è salito, adesso arriva all'altezza del bacino. Walter ha il binocolo davanti agli occhi, Carlo ha indossato una maglietta sgargiante e stringe le braccia al petto.

C. - Sto male. Fa caldo, c'è un'afa che stende. Sono scese due gocce e tu guarda: si sta peggio di prima... Io me ne vado.

W. - E aspetta. Inizia lo sport.

C. - C'è lo sport? (Impugna il canocchiale e si mette a guardare)

W. - Grande!

C. - Ti ricordi?

W. - Io ne ho viste... ma un'emozione così...

C. - Eravamo proprio qua.

W. - Il signor Bi aveva appena comprato il nuovo tv color.

C. - Dieci metri per venti di godimento assoluto. Lo sport in tv... come essere lì, a un metro dall'azione.

W. - Ai miei tempi stavi con un orecchio alla radio e via con l'immaginazione. L'immaginazione non porta niente di buono.

C. - (Smette di guardare la tv) Ti ricordi il centravanti? Come si chiamava?

W. - (Anche Walter smette di guardare la tv) Aveva quel nome strano...

C. - Ce l'ho sulla punta della lingua...

W. - Comunque fece un gol stupendo.

C. - Non hai caldo?

W. - È pazzesco, ma ci si fa l'abitudine.

C. - Si fa l'abitudine a tutto.

W. - Guarda un po' se c'è ancora lo sport?

C. - (Prende il binocolo e guarda la tv) No. Inizia il quiz.

W. - C'è quella presentatrice carina?

C. - Sì! Sì!

W. - (Prende il binocolo e guarda) Quanto mi piace.

C. - Sai una cosa?

W. - (Distratto) Cosa. 
Non mais t'y crois, toi?

C. - Fait chaud. Jamais vu un mois de juin aussi chaud.

W. -... Attends, attends... Nooon! Prends tes jumelles, il faut que tu voies ça...

C. - (Il prend ses jumelles avec nonchalance et regarde) Qu'est-ce qu'il y a?

W. - Le magicien! C'est dingue!

C. - Il a coupé son assistante en deux...

W. - (Plein d'admiration) Incroyable...

C. - Il l'a coupée en deux pour de bon!

W. - (comme précédemment) C'est un bon...

Noir. La lumière revient lentement. Carlo et Walter sont toujours au milieu de la rivière, le niveau de l'eau a monté, elle leur arrive maintenant à la hanche. Walter tient les jumelles devant ses yeux. Carlo a mis un t-shirt aux couleurs criardes et il serre ses bras contre sa poitrine.

C. - Je suis pas bien. Il fait chaud, une chaleur à tomber par terre. Il est tombé deux gouttes d'eau, et tu vois le résultat: on est plus mal qu'avant... Moi je m'en vais.

W. - Attends un peu. Il y a le sport qui commence.

C. - Le sport? (Il saisit les jumelles et se met à regarder)

W. - Joli!

C. - Tu te souviens?

W. - J'en ai vu pas mal... mais une émotion pareille...

C. - On était ici, justement.

W. - Monsieur Bé venait juste d'acheter sa nouvelle télé couleurs.

C. - Dix mètres sur vingt de jouissance absolue. Le sport à la télé... comme si on y était, à un mètre de l'action.

W.- De mon temps, on restait l'oreille collée à la radio, et fallait imaginer le reste. L'imagination n'amène rien de bon.

C. - (Il cesse de regarder la télé) Tu te rappelles l'avant-centre? Comment il s'appelait?

W. - (Walter cesse lui ausi de regarder la télé) Il avait un drôle de nom...

C. - Je l'ai sur le bout de la langue...

W. - En tout cas, il avait marqué un but formidable.

C. - T'as pas chaud?

W. - C'est dingue, mais on s'habitue.

C. - On s'habitue à tout.

W. - Regarde voir si c'est encore du sport? 
C. - (Lascia il binocolo) Ho i piedi ficcati nel fango e non mi riesce di muovermi.

W. - (C.s.) Sì ? Anch'io... Ohmmammamia... ohmmammamia... si è tolta il reggiseno... si è tolta il reggiseno $! . .$. se l'è rimesso...

C. - Non ci credo...

W. - (C.s.) Ti giuro!

C. - ... è incredibile, è come una morsa, non riesco a muovermi.

W. - (C.s.) Che roba... mammamia che roba...

C. - Mi ascolti!

W. - (Stizzito) E guarda la tv e lascia perdere. Ci penseremo poi ai tuoi piedi.

C. - È ancora nuda? (Impugna il binocolo e guarda)

W. - È stato un attimo: l'ha tolto e l'ha rimesso.. quelli del pubblico manca poco svenivano.

C. - Mi piacerebbe fare il pubblico una volta.. andare a una di queste trasmissioni.

W. - Basta telefonare.

C. - Magari conosco una di quelle ragazze.

W. - Ti piacerebbe, eh?... e quelle considerano uno come te. Ma ti sei visto?

Buio. La luce cresce lentamente, l'acqua è salita ancora. Carlo e Walter sono sommersi fino al collo. Walter continua imperterrito a guardare la tv con il suo binocolo.

W. - Fa due gocce e smette. O si decide a piovere o qua si schianta.

C. - Non pensi sia il caso di andare.

W. - Te fai che ti pare, io voglio vedere come va a finire.

C. - Almeno potremo cominciare a pensare...

W. - (Abbassa per un attimo il binocolo) Senti: lavoro tutto il giorno, ho i miei problemi, se almeno potessi guardare la tv in pace... Intesi?

C. - Sì, ma dovremo pensare a come uscire di qua. I miei piedi sono cementificati, i tuoi anche...

W. - Guardiamo la fine prima.

C. - E va bene... (Prende il binocolo e guarda) Lei è già stata dall'avvocato?

W. - Sì, ma l'ha cacciata.

C. - Perché?

W. - È tornata da lui a piangere come una bambina e lui l'ha cacciata.

C. - Ha fatto bene.

W. - Nessuna esitazione. 
C. - (Il prend les jumelles et regarde la télé) Non. C'est le début du jeu des questions.

W. - Avec la présentatrice qui est si bien roulée?

C. - Oui! Oui!

W. - (Il prend les jumelles et regarde) Elle me plaît drôlement.

C. - Tu sais quoi?

W. - (Distrait) Quoi?

C. - (Il lâche les jumelles) J'ai les pieds enfoncés dans la boue et je n'arrive pas à bouger.

W. - (comme précédemment.) Ah bon? Moi c'est pareil... Oh là là, oh ma mère, oh là là... elle a retiré son soutien-gorge... elle a retiré son soutien-gorge!... elle l'a remis...

C. - Je te crois pas...

W. - (comme précédemment) Je te jure!

C. - C'est incroyable, c'est comme un étau, j'arrive pas à bouger.

W. - (comme précédemment) C'est dingue... oh la vache, c'est dingue...

C. - Tu m'écoutes!

W. - (Agacé) Regarde donc la télé et laisse tomber. On y pensera plus tard, à tes pieds.

C. - Elle est encore nue? (Il saisit les jumelles et regarde)

W. - Ça n'a duré qu'un instant: elle l'a retiré et elle l'a remis... le public a failli s'évanouir.

C. - J'aimerais bien être dans le public, un jour... aller à une de ces émissions.

W. - Y'a qu'à téléphoner.

C. - Si ça se trouve, je pourrais faire la connaissance d'une des filles qui sont sur le plateau.

W. - T'aimerais bien, hein?... et tu penses qu'elles vont faire attention à un type comme toi? Non mais tu t'es vu?

Noir. La lumière revient lentement, l'eau a encore monté. Carlo et Walter sont dedans jusqu'au cou. Walter continue imperturbablement à regarder la télé avec ses jumelles.

W. - Il tombe deux gouttes et ça s'arrête. Si ça ne se décide pas à pleuvoir, on va crever.

C. - Tu crois pas qu'il faudrait y aller?

W. - Fais comme tu veux, moi je veux voir ce qui va se passer.

C. - On pourrait au moins commencer à penser à...

W.- (Il baisse un instant ses jumelles) Écoute: je bosse toute la journée, j'ai 
C. - Non ci posso credere!

W. - Eh... Visto? Tanto lo sapevo che andava a finire così.

C. - È il marito di sua sorella!!! Come può andare a letto con il marito di sua sorella!?

W. - Guarda che a mia cugina è successa la stessa cosa.

C. - Tua cugina non è la mia attrice preferita...

W. - Si sta consumando un dramma.

C. - E noi siamo qui, vorrei fare qualcosa...

W. - È la tv... Non ci puoi fare niente. Al limite puoi cambiare canale e far finta di non vedere.

C. - Noi non abbiamo il telecomando.

W. - Ringrazia che abbiamo' sto tecni-color tutto per noi.

C. - Fine. Meno male, non mi reggeva il cuore.

W. - Ehi, il signor Bi ha spento la tv...

C. - L'acqua è salita.

W. - Vabbé... Comunque è stata una bella serata.

C. - Sì.

\section{Pausa}

W. - Anche i tuoi piedi sono bloccati?

C. - Te l'avevo detto! Ma mi ascolti quando parlo?

W. - Va bene, va bene. Ci verrà in mente qualcosa. Pensiamo.

C. - Piove.

W. - Che venisse un bell'acquazzone una volta buona... Non è mai stato così caldo a giugno...

C. - Se continua così tra cinque minuti avremo la testa sott'acqua.

W. - Sei il solito disfattista. Pensa positivo, guarda avanti.

C. - A forza di pensare positivo non ti sei accorto di avere l'acqua fino al collo.

W. - Naturalmente è colpa mia.

C. - Sei stato incollato tutto il tempo alla tv.

W. - Perché te che hai fatto?

L'acqua è salita ulteriormente. Walter e Carlo sono costretti a tener ritta la testa per respirare.

W. - Si mette male.

C. - Non ti dovevo ascoltare.

W. - Senti, non è il momento di demoralizzarsi, se il fiume è salito, prima o poi scenderà, no? 
mes problèmes, alors si au moins je pouvais regarder la télé tranquille... Pigé?

C. - Oui, mais faudrait commencer à penser à la façon de sortir de là. Mes pieds sont coincés, les tiens aussi...

W. - On regarde la fin d'abord.

C. - Bon d'accord... (Il prend les jumelles et regarde) Elle est déjà allée chez l'avocat?

W.- Oui, mais il l'a jetée dehors.

C. - Pourquoi?

W. - Elle est revenue chez lui pleurnicher comme une gamine, et lui il l'a jetée dehors.

C. - Il a bien fait.

W. - Sans la moindre hésitation.

C. - J'y crois pas!

W. - Ah... tu as vu? De toutes façons, je le savais que ça se finirait comme ça...

C. - Mais c'est le mari de sa sœur!!! Comment peut-elle coucher avec le mari de sa sœur!?

W. - Tu sais, la même chose est arrivée à ma cousine.

C. - Ta cousine n'est pas mon actrice préférée..

W. - Nous sommes en train d'assister à un drame.

C. - Et nous on est ici, je voudrais pouvoir faire quelque chose...

W. - C'est la télé... On peut rien faire. A la limite, tu peux changer de chaîne et faire semblant de ne pas voir.

C. - Mais c'est pas nous qui avons la télécommande.

W. - Estime-toi heureux qu'on ait ces images en Technicolor rien que pour nous.

C. - Fin. Il était temps, ça me fendait trop le cœur.

W. - Eh là, Monsieur Bé a éteint la télé...

C. - L'eau a monté.

W.- Oui, bon... En tout cas on a passé une bonne soirée...

C. - Oui.

\section{Pause}

W. - Toi aussi t'as les pieds bloqués?

C. - Je te l'avais dit! Mais tu m'écoutes quand je parle?

W. - Ça va, ça va. On va trouver quelque chose. Réfléchissons.

C. - Il pleut.

W. - Si seulement il pouvait y avoir une bonne grosse averse, une fois pour toutes... Il fait jamais aussi chaud en juin... 
C. - Sì, allora potranno ritrovare facilmente i nostri cadaveri.

W. - Certo sei di grande consolazione, eh? (Con un guizzo di entusiamo)

Ha riacceso la tv!

C. - Sicuro?

W. - Ma sì! Ci sono i cartoni animati!

Carlo e Walter prendono il binocolo e cercano faticosamente di guardare, ma l'acqua è salita ancora.

C. - Se solo si potesse sentire.

L'acqua sale e copre completamente Walter e Carlo, le loro parole diventano un gorgoglio incomprensibile, coperto immediatamente dalla sigla, assordante, di un cartone animato. 
C. - Si ça continue comme ça, dans cinq minutes on aura la tête sous l'eau.

W.- Faut toujours que tu partes battu d'avance. Pense positif, regarde devant toi.

C. - À force de penser positif tu ne t'es pas rendu compte que tu avais de l'eau jusqu'au cou.

W. - Évidemment, ça va être ma faute.

C. - T'es resté collé tout le temps à la télé.

W. - Parce que toi, t'as fait quoi?

L'eau a encore monté. Walter et Carlo sont obligés de garder la tête bien droite pour respirer.

W. - Ça se gâte.

C. - J'aurais pas dû t'écouter.

W. - Ecoute, c'est pas le moment de se démoraliser, si la rivière a monté, tôt ou tard elle descendra, non?

C. - Oui, comme ça on pourra retrouver nos cadavres plus facilement.

$\mathrm{W}$. - Tu sais vraiment trouver les mots pour nous réconforter, hein? (Avec un éclair d'enthousiasme) Il a rallumé la télé!

C. - T'es sûr?

W. - Mais oui! C'est les dessins animés!

Carlo et Walter prennent leurs jumelles et essaient péniblement de regarder, mais l'eau a encore monté.

C. - Si seulement on pouvait avoir le son.

L'eau monte et recouvre complètement Walter et Carlo, leur mots se transforment en un gargouillis incompréhensible, immédiatement couvert par le gingle, assourdissant, d'un dessin animé. 